\title{
LA MIGRACIÓN, EL INGRESO Y EL EMPLEO URBANOS*
}

\author{
Manuel Gollás \\ El Colegio de México
}

\section{INTRODUCCIÓN}

EN MÉXICo el crecimiento acelerado del producto nacional no ha estimulado un aumento similar en el empleo. La fuerza de trabajo crece rápidamente, el desempleo urbano aumenta y, paradójicamente, se observa una fuerte corriente migratoria rural-urbana.

Se pueden distinguir en México dos mercados de trabajo: uno el tradicional y, otro, el del sector moderno de la economía. El salario en este último depende del ingreso medio del trabajador en el sector tradicional. Para compensar los costos monetario y no monetario de cambiar de sector, el salario en el sector moderno tiene que ser mayor que el del sector tradicional. Las diferencias de ingresos rurales y urbanos en México son muy marcadas. Una forma de medir las diferencias de salarios entre el sector tradicional y el moderno, es a través de las diferencias de productividad de la mano de obra en estos sectores. Se ha calculado que la productividad de la mano de obra en las áreas donde se localiza la agricultura de subsistencia es, en términos medios, la mitad de la de los trabajadores industriales no calificados en la industria. Así, no es aventurada la hipótesis de que un migrante típico de la agricultura tradicional recibirá, en el sector moderno, alrededor de un $50 \%$ más del ingreso que recibía en el sector de donde provino.

Una característica importante de la oferta de trabajo es su elasticidad, es decir, el aumento o disminución del número de trabajadores disponibles en respuesta a un cambio en el salario. Es razonable suponer que la oferta de trabajo a la que se enfrenta el sector moderno es elástica; si no lo fue-

* José Luis Estrada estuvo a cargo de la detectivesca tarea de encontrar los datos que se utilizaron en este estudio, así como de la elaboración y supervisión del programa estadístico empleado. Elena Espinoza de los Reyes colaboró en los diversos aspectos de la elaboración de este trabajo y Adalberto García Rocha hizo valiosos comentarios para mejorar la versión final. El autor expresa su agradecimiento a estas personas. Este trabajo fue financiado en parte por la Asociación Mexicana de Población. 
ra, los salarios habrían aumentado mucho más rápidamente de lo observado en el período de crecimiento acelerado cuando se demandó gran cantidad de mano de obra en el sector moderno. Con base en la evidencia empírica disponible, es difícil conocer el valor preciso de la elasticidad de la oferta de trabajo, pero se pueden hacer conjeturas. Si en la legislación de salarios mínimos, se hubieran fijado los salarios más arriba del nivel de equilibrio, entonces la oferta de trabajo hubiera sido perfectamente elástica a ese nivel de salario. Esto no ha ocurrido en México. La productividad media real del sector tradicional ha aumentado, y esto quiere decir que el sector moderno no se enfrentó a una oferta de trabajo perfectamente elástica. Es decir, a medida que parte de la fuerza de trabajo del sector tradicional dejó el campo y emigró al sector urbano, aumentó el producto medio de la mano de obra que permaneció ahí, y fue necesario pagar un salario más elevado en el sector moderno para atraer a la nueva fuerza de trabajo. Aun cuando la evidencia no es definitiva, apoya la hipótesis de que la oferta de trabajo al sector moderno depende del salario que se pague en este sector.

Veamos ahora algunos aspectos de la demanda de mano de obra en México. De manera específica, el efecto que ha tenido el aumento del producto sobre el empleo. Para ello es necesario conocer los determinantes y la magnitud de la elasticidad-producto del empleo, o sea, el cambio relativo en el empleo causado por un aumento relativo en el producto.

Una manera de estimar la elasticidad del empleo, es mediante funciones de demanda de trabajo, que se derivan a su vez de una función de producción sin economías de escala, o de otras versiones más elaboradas de la función de producción. Antes de mencionar los cálculos que se han hecho de la elasticidad empleo-producto en México, se harán algunas reflexiones sobre este concepto en relación con el desempleo.

Es un hecho aceptado que la fuerza de trabajo en México se duplicará en un lapso no mayor de 20 años y que es necesario encontrarle trabajo. Siendo éste el caso, pudiera parecer extraño el objetivo de política económica de elevar la elasticidad empleo-producto, o sea, detener el crecimiento de la productividad que a su vez implića una economía estacionaria. ${ }^{1}$ El razonamiento no es erróneo porque la elevación de la productividad se aplica a economías desarrolladas, y no a aquellas en proceso de crecimiento, como la mexicana. Veamos hipotéticamente los casos extremos de un país desarrollado y otro en desarrollo.

Supóngase que en un país desarrollado el nivel de empleo es siempre el de empleo pleno. Si es así, el nivel de empleo está determinado exógenamente por el tamaño de la fuerza de trabajo, y la tasa de salarios fluctúa libremente. Supóngase ahora un país en desarrollo donde ocurre precisamente lo contrario, o sea, donde el salario se fija de manera exógena a nivel de subsistencia y el nivel de empleo fluctúa libremente.

1 Se considera estacionaria cuando la productividad marginal del trabajo no aumenta. 
En estas condiciones, cuando en un país desarrollado aumenta el acervo de capital y la tecnología avanza, el ingreso y la producción aumentan. El aumento en la producción, el capital y la tecnología elevan la demanda de mano de obra. Pero, como la oferta de trabajo no puede responder a esta situación porque es fijada de manera exógena, el salario real aumenta para establecer el equilibrio en el mercado de trabajo. Así, en este caso, el desarrollo económico aumenta el producto por trabajador y el nivel de salarios. El aumento del producto por trabajador (productividad del trabajo) es entonces un indicador del desarrollo de la economía.

Veamos el caso de un país en desarrollo. La acumulación de capital y el desarrollo tecnológico también hacen, como en el caso del país desarrollado, que el ingreso aumente. En este caso, la elevación de la demanda de trabajo se puede realizar, no mediante un cambio en los precios relativos de los factores, como en el caso anterior, sino a través de un aumento en el empleo que es la variable libre de fluctuar. Entre más rápido sea el crecimiento del capital y el desarrollo tecnológico, más rápido puede ser el crecimiento del empleo. En estas condiciones la productividad no aumenta, o lo hace de manera lenta, pero esto no indica estancamiento. En un país subdesarrollado con elevados niveles de desempleo, estancamiento sería mantener un nivel constante de empleo con salarios más elevados. Una elasticidad empleo-producto elevada sí implica que la productividad de la mano de obra crece con lentitud, pero esto no debe resultar extraño.

En México el rápido crecimiento del producto no ha estimulado un crecimiento similar del empleo. Se ha calculado que durante el decenio 19501960, el producto no agrícola de México creció a una tasa dos veces mayor que el empleo de ese sector. Una relación semejante se observó en el período 1954-1960 en el sector industrial. También se han hecho otras estimaciones y se encontró que, aun cuando el crecimiento del empleo en el sector industrial fue considerable (3.8\% anual entre 1954-1960 y $4.3 \%$ anual entre 1950-1960), este crecimiento fue notablemente menor (la mitad) que el del producto en este sector. Para el período 1959-1975, la tasa de crecimiento anual de la ocupación en este sector fue de $3.8 \%$. Luego el sector industrial ha disminuido recientemente su capacidad de generar nuevos empleos. La elasticidad producto-empleo, de los subsectores que componen el sector no agrícola, varía entre 0.4 y 0.6 . La elasticidad es pues positiva, pero muy baja.

Otro aspecto central de una política de empleo es conocer los efectos cuantitativos de un cambio en el producto y en los salarios sobre el empleo. El México se ha encontrado (Isbister, 1969) que el efecto de un aumento en el nivel de salarios sobre el empleo es negativo. El efecto es relativamente débil en la preparación de alimentos y productos industriales de vidrio, pero en otras industrias es bastante más acentuado. Se analizaron series de tiempo y se encontró que la elasticidad del empleo en relación a los salarios es negativa y significativamente mayor que 1 en términos absolutos. Resultados similares se encontraron al utilizar datos de corte transversal. 
Los salarios en el sector moderno de México han aumentado lentamente y el empleo relativamente más rápido. Así pues, los salarios en México tienen un efecto importante sobre el empleo.

La situación que se presenta en México es como sigue: La población ha crecido durante los últimos años a la elevada tasa de $3.5 \%$ al año, lo que hará que la población se duplique en 20 años. Entre 1950 y 1960 el empleo en el sector moderno no agrícola creció aproximadamente a la mitad de lo que creció el producto. En el sector manufacturero, el empleo creció a más o menos la mitad de lo que creció el producto en ese sector entre 1954-1966 y entre 1969-1975. En este mismo sector, la elasticidad empleo-producto en el largo plazo se estimó en aproximadamente 0.8. El aumento relativamente modesto de los salarios que ocurrió durante 1954-1966 redujo esta elasticidad en 25 industrias, durante el período 1953-1967, donde se encontró una elasticidad empleo-producto de 0.84 y una elasticidad salario-empleo de -0.52 . Por último, la corriente mi-

- gratoria rural-urbana durante los últimos 30 años ha aumentado con gran

- rapidez a pesar del creciente desempleo urbano. En la sección II de este artículo, se presenta una revisión de la literatura económica y de los mo-

- delos que usa para explicar la migración rural-urbana y en la sección III se emplea uno de estos modelos con el fin de encontrar algunas de las causas de la migración rural-urbana en México.

\section{- II. LoS MODElos DE MigRAción y LOS OBJETIVOS DEL ESTUdio}

Se ha encontrado que entre 1950 y 1960 más de 2.2 millones de personas cambiaron de residencia cuando menos una vez, y que la mayor parte de la migración interna en México ocurre en forma de migración rural-urbana (Ehitten y Burnight, 1956). En este mismo estudio se encontró que casi el $90 \%$ de la migración interestatal entre 1940 y 1950 vivía en zonas urbanas. Asimismo, el desempleo en estas zonas es muy grande. Las explicaciones más comunes sobre el porqué el sector urbano no ha podido dar empleo a la migración rural son: a) los precios deformados de los factores que dan lugar a un sesgo en los métodos de producción hacia el uso de tecnologías intensivas en capital; $b$ ) las tarifas y otros mecanismos de comercio internacional que con frecuencia resultan en tasas negativas de protección para bienes de capital importados y que, en conjunción con una política liberal de impuestos sobre la depreciación del capital, hacen que el costo del capital sea menor que su costo de oportunidad; $c$ ) la posición cada vez más poderosa de los sindicatos y la legislación de salarios del gobierno, han logrado un salario urbano industrial significativamente más elevado que el costo de oportunidad de la fuerza de trabajo urbano. En estas circunstancias, es de esperarse que las empresas adopten, de manera deliberada, técnicas mecanizadas de producción. 
La combinación de una creciente población urbana que aumenta debido a la migración, y un lento crecimiento del empleo urbano, ha dado lugar a una creciente reserva de trabajadores urbanos desempleados.

Existen numerosas teorías para explicar los movimientos migratorios del campo a la ciudad. Entre éstas podemos mencionar la de Zipf (1964) y la de Stouffer (1940) que se basan en los modelos de gravedad y la distancia entre los puntos de migración. En estos modelos el interés han sido las características de atracción y repulsión en los lugares de origen y de destino. Estos modelos son análogos al modelo newtoniano en la física. En términos generales, se postula que la atracción de dos lugares, observable mediante el flujo de migración entre ellos, puede definirse como una relación inversa a la distancia o, de manera más simple, que la tasa total de migración entre dos puntos se atenúa en una proporción que varía con el cuadrado de la distancia entre ellos. Otros modelos para explicar la migración son los que se han dado en llamar "Push-Pull". En estos modelos se consideran las fuerzas expulsivas del lugar de origen y las de atracción de las de destino. En los modelos de "Push-Pull" se concede especial atención al estudio de los factores que hacen que las personas abandonen las áreas pobres que no crecen y emigren a las más ricas en expansión. Una versión particular de este tipo es la de Stouffer (1940), quien conjetura que la migración entre dos lugares es directamente proporcional a las diferencias de oportunidades económicas, e inversamente proporcional al número de oportunidades que compiten entre la distancia que los separa. En estudios de Stollman y de Ball (1971) para México se ha encontrado que las condiciones económicas explican menos del $50 \%$ de la variación en las tasas de migración entre los municipios del país.

Otros modelos más complicados son los modelos probabilísticos. Uno de los más comunes utiliza el concepto de las cadenas de Markov y predice la distribución de la población futura sobre un campo de migración con base en una matriz de probabilidades de transición.

En el estudio presente se siguió un procedimiento diferente al de los modelos descritos, cuya orientación es principalmente demográfica y no incorpora de manera explícita hipótesis derivadas de la teoría económica.

Se hace ahora una breve exposición de otras dos corrientes mediante las cuales se trata de explicar la migración rural-urbana: la primera utiliza el concepto de inversión en capital humano, y la segunda incorpora restricciones probabilísticas a estas nociones. La teoría del capital humano es adecuada para explicar la dirección de la migración y, hasta cierto punto, para explicar qué tipo de personas emigran. La conclusión lógica de esta corriente es que las diferencias salariales que explican la migración eventualmente desaparecen y, por lo tanto, también la migración. Esta predicción obviamente no corresponde a los hechos observados. La corriente explica la migración como una función probabilística de encontrar empleo en el sector urbano, admite la posibilidad que se observe fuerte 
migración a pesar del crecimiento del desempleo urbano. Las características más sobresalientes de estas corrientes se explican a continuación.

\section{La orientación de capital humano}

Conforme a esta corriente se considera a la migración como una inversión en capital humano; una inversión que tiene costos, pero de donde también se obtienen ganancias. La migración se realiza si el migrante potencial percibe que la diferencia entre el valor presente de los ingresos futuros de dos lugares es más grande que los costos de migración. Los ingresos y los costos son monetarios y no monetarios. Los ingresos monetarios pueden medirse como la diferencia entre ingresos monetarios esperados en los dos lugares. Los costos monetarios son los gastos que se necesitan para trasladarse y buscar empleo. Los costos no monetarios son las ganancias que se han dejado de percibir mientras se viaja. La regla de decisión, desde el punto de vista del modelo de capital humano, consiste en comparar los flujos de ganancias capitalizadas entre las actividades de migración y no migración, considerado el valor de la tasa de interés y la duración de cada actividad.

Se puede expresar el modelo general de capital humano para el caso discreto mediante la siguiente fórmula:

$$
\stackrel{\Sigma}{t}_{\underline{\underline{\Sigma}}}^{m} \frac{W(t)}{(1+r)^{t}}-C(0)-\underbrace{m}_{t \underline{\Sigma}_{0}} \frac{R(t)}{(1+r)^{t}}>0
$$

En esta expresión $W$ es el salario anual esperado en el sector urbano; $R$ el ingreso anual en el empleo rural; $C$ el costo de la migración; $m$ el número de años que espera vivir el individuo en la zona urbana; y $r$ una tasa de interés. Como se señaló, el costo de la migración incluye los siguientes componentes: los costos directos que son el costo de transporte y traslado; el costo indirecto de oportunidad, que es el ingreso que se deja de ganar mientras se viaja; y el gasto en el que se incurre al buscar trabajo.

Para el caso continuo se tiene:

$$
\begin{aligned}
& V_{U}(O)=\underset{t=0}{s} W(t) \exp (-r t) d t-C(O) \\
& V_{R}(O)={ }_{t=0}^{m} R(t) \exp (-r t) d t
\end{aligned}
$$

En estas expresiones $V_{U}$ y $V_{R}$ son los valores presentes de los ingresos urbanos y rurales. En esta forma el salario urbano y el ingreso rural son ahora función del tiempo. Se puede ahora escribir la diferencia urbanorural:

$$
\alpha=V_{U}(O)-V_{R}(O)
$$


Donde $\propto$ es función del flujo esperado de ingreso, de $W$ y $R$, de los costos de migración $C$, del periodo de trabajo esperado $m$, y de la tasa de interés $r$ que se aplicará en cada caso.

La interpretación de este modelo es simplemente que la migración está determinada por $\alpha$, que es el valor capitalizado de la diferencia neta entre los flujos de ingreso rural y urbano.

Este modelo explica ciertos hechos de la migración. Predice de manera correcta que la migración se lleva a cabo de las áreas rurales de bajo ingreso a las regiones urbanas de más elevados ingresos. Esto se debe a que los costos directos de migración varían según la distancia. El modelo de capital humano predice que la migración puede realizarse en etapas: de la parcela al pueblo, del pueblo a la ciudad y, tal vez, de la ciudad al extranjero. Este modelo también señala que la gente joven es la que emigra. El efecto edad está representado por $m$, que es mayor para la gente joven, la cual tiene una mayor esperanza de vida para capitalizar las diferencias en ingreso al migrar de un lugar a otro. Por otro lado, la gente joven es por lo general más pobre, lo que hace que en el lugar de origen $R$ sea más reducido. También ocurre que los jóvenes tienen menos arraigo en el lugar de origen, lo cual implica que $C$ sea reducido. $C$ relativamente se reduce aún más porque la mayor parte de los jóvenes son solteros.

El modelo de capital humano aplicado a la migración ha sido verificado por Sjaastad (1962), Bowles (1970), Fabricant (1970), Galloway, Gilbertz, Smith (1967), Greenwood (1969), Laber y Chasse (1971), Lowry (1966), Miller (1973), Rogers (1967), Sommers (1973), Wadycki (1974), en los Estados Unidos; y por Courchene (1970) en Canadá. En América Latina ha sido usado por Sahota (1968) para el caso de Brasil; por Herrick (1965) en Chile; y por Schultz (1971) en Colombia. En estos estudios el modelo ha explicado de manera satisfactoria algunos aspectos importantes del fenómeno migratorio.

\section{El modelo probabilístico de migración rural-urbana y empleo}

Este modelo (Todaro, 1970, 1971), (Gugler, 1969), modifica al del capital humano de tal forma que la diferencia urbano-rural es, de manera simultánea, determinante de la oferta de mano de obra al sector urbano, y función del nivel de desempleo urbano. El énfasis de este modelo se asigna en los costos indirectos de migración, en especial el que se refiere al salario que se deja de percibir mientras se busca trabajo en el sector urbano. Esta consideración es de particular importancia en situaciones de desempleo severo en las ciudades, lo cual sucede en la mayoría de ellas. Este modelo explica por qué la decisión de emigrar es una elección racional, a pesar de que las probabilidades de encontrar trabajo urbano sean pequeñas en el corto plazo. Por ejemplo, aun cuando la tasa de desempleo urbano sea bastante elevada ( 30 a $50 \%$ ), en principio esto no debe ser obstáculo para que la migración se realice si el ingreso en los centros 
urbanos excede al rural entre 300 a $400 \%$. Para explicar de manera satisfactoria la migración deben considerarse no sólo las diferencias absolutas entre los ingresos urbanos y rurales, sino que estas diferencias deben ajustarse o deflacionarse, con la probabilidad de encontrar trabajo urbano. Es en esta forma como el ingreso diferencial esperado se convierte en la variable que explica la decisión de emigrar. Mientras el ingreso urbano esperado exceda al ingreso rural esperado, el migrante está optando por una decisión económica racional si deja el campo y busca trabajo en un área urbana, a pesar de que sus oportunidades de encontrar trabajo en el corto plazo sean limitadas. Aún más, entre mayor sea su capacidad de sobrevivir cuando está buscando trabajo, ya sea por la ayuda de familiares o de amigos, más tiempo estará dispuesto a estar desempleado con la esperanza de encontrar un trabajo urbano bien pagado.

Si se supone por ejemplo que la diferencia de ingreso rural-urbano neto es de $\$ 100$, y si la probabilidad de encontrar trabajo urbano es sólo de $50 \%$, la diferencia urbano-rural de ingreso es realmente menor. Conforme a Todaro (1970-1971), esta diferencia ajustada $(\alpha)$ puede escribirse como:

$\alpha=\pi W-R$

Donde $W$ y $R$ son los salarios urbanos y rurales respectivamente, ambos netos de gastos de migración, y $\pi$ es la probabilidad de encontrar trabajo en el sector urbano en un periodo dado de tiempo.

Por otro lado, la probabilidad de encontrar trabajo en el sector urbano es función de la magnitud del desempleo y de la tasa de creación de empleo en ese sector. Se tiene así que:

$$
\pi=\frac{\delta N}{S-N}=\frac{\delta(1-d)}{d}
$$

Donde $N$ es el nivel de empleo urbano; $S$ el tamaño de la fuerza de trabajo urbana; $\delta$ la tasa neta de creación de empleo urbano; y $d$ la tasa de desempleo en el medio urbano. Al sustituir (2) en la ecuación (1) se puede redefinir la diferencia rural-urbana de ingreso como:

$$
\alpha=\frac{W \delta N}{S-N}-R
$$

Se puede ver que conforme a esta orientación, la oferta de trabajo de los migrantes al sector urbano, $S$, se considera función de la diferencia rural-urbana de ingreso:

$$
S=f_{S}(\alpha) ; \frac{\partial S}{\partial \alpha}>0
$$

Desde el punto de vista de la demanda de mano de obra, $D$, la tasa de creación de empleo urbano puede expresarse como función del salario 
urbano, $W$, y de un parámetro $k$ que representa la política del gobierno para aumentar el empleo mediante algún programa, por ejemplo, de sustitución de importaciones.

Así, se tiene:

$$
\delta=f_{D}(W, R) ; \frac{\partial \delta}{\partial W}<0 ; \frac{\partial \delta}{\partial R}>0
$$

Si la demanda de mano de obra urbana aumenta como resultado de algún programa de gobierno, el aumento de la oferta de mano de obra al sector urbano puede expresarse de la siguiente forma:

$$
\frac{\partial S}{\partial R}=\frac{\partial S}{\partial \alpha} \frac{\partial \alpha}{\partial \delta} \frac{\partial \delta}{\partial R}
$$

Al diferenciar (3) y sustituir en (6), se tiene:

$$
\frac{\partial S}{\partial R}=\frac{\partial S}{\partial \alpha} W \frac{N}{S-N} \frac{\partial \delta}{\partial R}
$$

Es claro que el desempleo urbano aumentará si el aumento en la oferta de mano de obra excede el número de empleos urbanos, esto es, si ocurre que:

$$
\frac{\partial S}{\partial R}>\frac{\partial(\delta N)}{\partial R}=\frac{\partial \delta N}{\partial R}
$$

Si se sustituye (7) en (8) se obtiene:

$$
\begin{aligned}
& \frac{\partial S}{\partial \alpha} W \frac{N}{S-N} \cdot \frac{\partial \delta}{\partial R}>\frac{\partial \delta N}{\partial R} \\
& \therefore \frac{\frac{\partial S}{S}}{\frac{\partial \alpha}{\alpha}}>\frac{\alpha}{W} \frac{(S-N)}{S}
\end{aligned}
$$

y sustituyendo por $\alpha$

$$
\frac{\frac{\partial S}{S}}{\frac{\partial \alpha}{\alpha}}>\frac{W \cdot \Pi-R}{W} \cdot \frac{(S-N)}{N}
$$


Las ecuaciones (10) y (11) expresan las condiciones de un desempleo urbano creciente en términos de la elasticidad de la oferta de mano de obra relativa a la diferencia expresada de ingreso rural-urbano. La elasticidad $\frac{\partial S}{S} / \frac{\partial \mathscr{Q}}{\alpha}$ puede considerarse como "una función de respuesta de la migración, o sea, la elasticidad de la oferta de trabajo rural en relación a la diferencia del salario urbano-rural descontado por la probabilidad de encontrar trabajo en el sector urbano.

El nivel absoluto de desempleo aumentará mientras la "función de respuesta de la migración" $\frac{\partial S}{S} / \frac{\partial \alpha}{\alpha}$ exceda a la diferencia urbano-rural expresada ésta como una proporción del salario urbano $W$ multiplicado por la tasa de desempleo $\frac{S-N}{S}$.

Este modelo explica por qué las diferencias de salarios entre el campo y la ciudad persisten a pesar de un creciente desempleo urbano. Puede explicar, también, por qué una política de orientación principalmente urbana para disminuir el desempleo urbano hace que éste aumente. Sin este tipo de políticas el desempleo urbano actuaría como un factor que reduciría la migración. Sin embargo, estas políticas urbanas hacen que el desempleo urbano no tenga este efecto ya que, a pesar del aumento en desempleo urbano, la diferencia de ingreso rural-urbana continúa ampliándose debido a factores institucionales y políticos. De esta manera se neutraliza el efecto de la diferencia de ingreso esperado y continúa el flujo migratorio de las zonas rurales a las urbanas. Así, mientras la diferencia de ingreso esperado continúa en favor del sector urbano, aun un programa acelerado de creación de empleos en las áreas urbanas tendrá poco efecto sobre el desempleo, y tal vez hasta lo acentúe más.

Todo depende de la magnitud de la "función de respuesta de la migración". Para formular programas de empleo es importante saber cuántos campesinos se trasladarán a los centros urbanos como resultado de la creación de cada nuevo trabajo que se crea en éstos. Si este modelo es correcto, se deduce entonces que la solución al desempleo urbano no consiste en crear empleos urbanos, sino en estimular el desarrollo rural de manera de tener más empleos en este sector.

Las conclusiones de este modelo, de ser correctas, invalidarían las formulaciones que insisten en soluciones urbanas al problema del desempleo y también pondría en duda la eficacia de programas para aumentar el empleo por la acción directa o indirecta del Estado mediante subsidios a empresas urbanas.

\section{Migración, IngReso y DESEMPLEO}

En esta sección se someten a prueba las hipótesis sobre la contribución relativa de las diferencias de ingresos urbanos-rurales y las oportunidades 
de empleo urbano como factores explicativos de la migración al área metropolitana de la ciudad de México (AM).

\section{Introducción}

La migración rural-urbana es la más importante de la migración interna en el país y dentro de ésta destaca la corriente que se dirige hacia el área metropolitana de la ciudad de México. Según la encuesta sobre "Migración interna, estructura ocupacional y movilidad social en el área metropolitana de la ciudad de México" (EMICM), realizada por El Colegio de México (cubre el período 1960-1970), se encontró que cerca del 36\% del aumento demográfico en este período se debe a la migración.

Por otra parte, se ha calculado que el $90 \%$ de los migrantes al área metropolitana de la ciudad de México provienen de las cuatro grandes regiones que la circundan: la Centro-Oriente, la Periferia urbanizada, la Periferia no urbanizada y la Centro-Occidente que comprenden los estados de Hidalgo, parte del Estado de México, Morelos, Puebla, Tlaxcala, Guanajuato, Michoacán, Querétaro, San Luis Potosí, Veracruz, Guerrero, Oaxaca, Jalisco, Colima, Nayarit, Aguascalientes y Zacatecas (Muñoz, de Oliveira, Stern, 1979).

Respecto a la evolución de los salarios, la información publicada de la Comisión Nacional de Salarios Mínimos (CNSM), para 1950-1970, muestra que el salario mínimo establecido para el AM no sólo es mayor que el correspondiente para el medio rural sino que dicha diferencia aumentó. Por desgracia, se carece de información sobre el crecimiento regional de los precios para este período ya que la CNSM ha elaborado un índice de precios sólo a partir del año de 1965. Por tal motivo no fue posible cuantificar el crecimiento real de los salarios mínimos.

Sin embargo, otros estudios señalan algunas conclusiones sobre los ingresos y salarios que pueden ser utilizadas en este estudio:

a) El análisis del crecimiento de los precios entre 1940 y 1970 puede dividirse en dos períodos (Trejo, 1978). El primero, 1940-1958, estuvo caracterizado por un proceso inflacionario acelerado conforme al cual se observa hasta 1950 una disminución de la participación de los salarios en el ingreso nacional.

En el segundo período, 1958-1970, se observó una mayor estabilidad de los precios y la participación de los salarios en el ingreso nacional creció en forma moderada. Así, es razonable suponer que para el período de estudio hubo un crecimiento real del ingreso personal.

b) Respecto al salario medio industrial, se ha calculado (Isbister, 1969), que registró un aumento del $50 \%$ entre 1950 y 1964 y, por otra parte, que la legislación de salarios mínimos no afecta de manera sensible la determinación del ingreso en el sector moderno.

c) El análisis de las declaraciones de ingresos de migrantes al AM durante los decenios de 1950 y 1960 , muestra que perciben un salario alrededor de 
1.7 veces mayor al fijado por la CNSM. Se ha encontrado también que el ingreso de los mirantes aumenta con el nivel de calificación: para 1970 el ingreso de los trabajadores que se incorporaron a la población económicamente activa de la ciudad de México como obreros no calificados de la industria de bienes de producción era alrededor de dos veces mayor al mínimo legal (de Oliveira, García, Muñoz, 1979).

De lo anterior puede inferirse que, en el período de estudio, aumentó la diferencia de ingresos personales entre las áreas rurales y urbanas.

\section{El modelo y sus variables}

Cabe señalar que en este modelo las variables de estudio son las diferencias de ingresos entre el medio urbano y el medio rural y no sus valores absolutos.

La diferencia de ingreso puede definirse de varias maneras. La primera y más simple es

$$
\alpha(t)=Y_{U}(t)-Y_{R}(t)
$$

en donde:

$\alpha(t)=$ diferencia entre ingresos familiares urbano y rural;

$Y_{U}(t)=$ ingreso medio urbano en el año $t$;

$Y_{R}(t)=$ ingreso medio rural para el año $t$.

La segunda definición de la variable diferencial de ingreso es:

$$
\alpha_{2}(t)=\sum_{j=1}^{N t} \frac{Y_{U}^{j}(t)-Y_{R}^{j}(t)}{(1+r)^{j}}
$$

en donde:

$\alpha_{2}(t)=$ diferencia descontada de ingresos entre el medio urbano y rural para el año $t$;

$Y_{U}^{j}(t)=$ ingreso esperado por el migrante en el año $t$, para el año $t+j$ en el medio urbano;

$Y_{R}^{j}(t)=$ el ingreso esperado por el migrante en el año $t$, para el año $t+j$ en el medio rural;

$r=$ tasa de descuento que refleja las preferencias de consumo y ahorro del migrante;

$N_{t}=$ indicador de la esperanza de vida activa de los migrantes del año $t$ en el medio urbano.

En la definición $\alpha$, se mide la diferencia de ingreso corriente y en $\alpha_{z}$ se mide la diferencia descontada del flujo de ingresos. 
La migración es entonces función de la diferencia de salarios de acuerdo a las dos definiciones de $\alpha$ :

$$
\begin{aligned}
& \dot{M}(t)=M\left(\alpha_{1}\right) \\
& M(t)=M\left(\alpha_{2}\right)
\end{aligned}
$$

Para este estudio se hicieron cálculos de estas funciones con información de series de tiempo y de corte transversal.

Los resultados obtenidos son los siguientes:

a) Serie de tiempo según la definición $\alpha_{2}$.

El período analizado es de 1950 a 1970 . Los resultados según las especificaciones semilogarítmicas y logarítmicas son

$$
\begin{aligned}
& L M=8.22+0.00016 \alpha_{2}(0.00003) \\
& R^{2}=0.567 ; F(1,18)=23.62 \\
& L M=-9.16+2.052 L \alpha_{2} \\
& \quad(0.444) \\
& R^{2}=0.543 ; F(1,18)=21.36
\end{aligned}
$$

en donde:

$$
\begin{aligned}
& L M=\text { logaritmo de la migración; } \\
& L \alpha_{2}=\text { logaritmo de } \alpha_{2}
\end{aligned}
$$

b) Serie de tiempo, según la definición $\alpha_{1}$.

$$
\begin{aligned}
L M & =9.22+0.00118 \alpha_{1} \\
& (0.00028) \\
R^{2} & =0.503 \quad ; F(1,18)=18.24
\end{aligned}
$$

En las tres ecuaciones anteriores $(14,15$ y 16$)$ los coeficientes son significativos y el ajuste es bastante bueno (los valores de $R_{2}$ son elevados). El mejor ajuste corresponde a la regresión logarítmica con $\alpha_{2}$ que mide la diferencia del flujo descontado de ingresos.

En la formulación logarítmica (regresión 15) el coeficiente de $\alpha_{2}$ puede interpretarse como la elasticidad de la migración respecto a la diferencia de ingreso rural-urbano descontada. En la especificación semilogarítmica, la elasticidad es 0.00016 por $\alpha_{2}$ es decir, la elasticidad es función lineal del coeficiente de regresión.

La regresión 15 indica que la corriente migratoria aumenta más que proporcionalmente ante incrementos en la diferencia del ingreso rural urbano. Según esta ecuación, un aumento de $100 \%$ en la diferencia rural- 
urbana más que duplica el número de migrantes al área metropolitana de la ciudad de México.

Los cálculos respecto de la migración al área metropolitana de la ciudad de México por regiones específicas de origen son los siguientes.

a) Estimaciones de acuerdo con información de series de tiempo, y según la definición $\alpha_{1}$ :

Región 1 (Baja California Norte, Baja California Sur, Sinaloa, Sonora y Durango)

$$
\begin{aligned}
& L M R 1= 6.524+0.02284 \alpha_{1} R 1 \\
&(0.00790) \\
& R^{2}=0.433 ; F(1,18)=8.41
\end{aligned}
$$

en donde

$L M R 1$ = logaritmo del flujo migratorio de la región I al área metropolitana.

Región 2 (Jalisco, Colima, Nayarit, Aguascalientes y Zacatecas)

$$
\begin{gathered}
L M R 2=-7.173+0.01101 \alpha_{1} R 2 \\
(0.0036) \\
R^{2}=0.347 ; F(1,18)=9.03
\end{gathered}
$$

Región 3 (Guadalajara, Michoacán, Querétaro, San Luis Potosí y Veracruz)

$$
\begin{gathered}
L M R 3=8.659+0.00904 \alpha_{1} R 3 \\
(0.0023) \\
R^{2}=0.453 ; F(1,18)=14.89
\end{gathered}
$$

b) Las estimaciones de acuerdo con información de series de tiempo y según la definición $\alpha_{2}$, sólo fueron significativas para la región 2:

$$
\begin{aligned}
& L M R 2= 4.76+0.00146 \alpha_{2} R 2 \\
&(0.00044) \\
& R^{2}=0.388 ; F(1,18)=19.76
\end{aligned}
$$

De acuerdo con los resultados de la definición $\alpha_{1}$, para cada valor de la diferencia entre el ingreso rural-urbano, la migración al área metropolitana de la ciudad de México será mayor de la región 1 que de la región 2 o la 3.

Las regresiones para las otras regiones ( 4 y 5 ) en los que se dividió la república no resultaron significativas. 


\section{Análisis de corte transversal}

Las pruebas de corte transversal efectuadas no dieron resultados satisfactorios. La correlación entre la migración y las diferencias de ingreso fue baja. Debe señalarse que en el análisis de corte transversal se consideró a la distancia como una variable en el costo de migrar.

Para los cuatro quinquenios comprendidos entre 1950 y 1969 se realizaron pruebas de corte transversal donde la variable dependiente es el volumen migratorio de la entidad federativa $i$ para el quinquenio correspondiente. Las variables independientes fueron la diferencia de ingreso y el costo de migración, el cual se consideró determinado principalmente por la distancia.

Como ejemplo, se presentan los resultados con mayor grado de ajuste. Sin embargo, ninguno de ellos merece comentarios por el nivel tan reducido de los $F$ obtenidos.

$$
\begin{gathered}
L M Q 2=5.141-0.00141 D+0.00018 \alpha_{1} Q 2 \\
(0.00051)(0.00015) \\
R^{2}=0.176 ; F(1,23)=1.57 \\
L M Q 1=5.04-0.00187 D+0.00005 \alpha_{2} Q 1 \\
R^{2}-0.181 ; F(1,22)=1.43
\end{gathered}
$$

en donde:

$$
\begin{aligned}
L M Q 2= & \text { logaritmo de la migración en el segundo quinquenio } \\
L M Q 1= & \text { logaritmo de la migración en el primer quinquenio; } \\
D= & \text { distancia, en kilómetros, de la capital de la entidad al área } \\
& \text { metropolitana. }
\end{aligned}
$$

\section{El modelo cuando se considera la probabalidad de encontrar empleo}

Conforme a esta especificación de la diferencia de ingreso rural-urbano se considera la probabilidad de que el migrante encuentre trabajo en el sector urbano. Al hacer esto, las decisiones del migrante dependen del valor esperado del ingreso actualizado, en general menor al ingreso en el caso de certidumbre absoluta. Así, se tiene una nueva definición de $\alpha$ :

$$
\alpha_{33}(t)=\sum_{j=1}^{N_{t}} \frac{Y_{u j}(t) P_{j}(t)-\bar{Y}_{j}(t)}{(1+r)^{j}}
$$

en donde:

$\alpha_{3}(t)=$ valor de la diferencia de ingreso rural-urbano; 
$Y_{u j}(t)=$ ingreso anual esperado por el migrante en el área metropolitana en el año $t+j$;

$\bar{Y}_{j}(t)=$ ingreso medio esperado por el migrante para el año $t+j$ en las zonas rurales de los estados de mayor emigración al área metropolitana;

$P_{j}(t)=$ probabilidad de que el migrante que llegó al $A M$ en el año $t$ tenga trabajo en el "sector moderno" en el año $t+j{ }^{2}$

$N_{t}=$ indicador de la esperanza de vida de los migrantes para el año de migración $t$.

$r=$ tasa de descuento.

Los resultados que se obtuvieron según esta formulación son:

$$
\begin{gathered}
L M=8.084+0.000016 \alpha_{3}(t) \\
(0.000003) \\
R^{2}=0.545 ; F(1,18)=21.57 \\
L M=-15.435+2.175 L \alpha_{3}(t) \\
(.494)=19.4
\end{gathered}
$$

Estos resultados pueden interpretarse como sigue. Cuando se considera la diferencia de ingreso rural-urbano como causante de la migración se tiene en cuenta el hecho de que el migrante no encuentre trabajo por un tiempo $\left[P_{j}(t)\right]$, se observa que el efecto de esta diferencia de ingreso sobre la migración es considerable. Según la regresión 25 un aumento de $100 \%$

2 La probabilidad de que el migrante que llegó al área metropolitana en el año $t$ se encuentra trabajando en el sector moderno en el año $t+i$ puede expresarse como

$$
P_{j}(t)=P_{j-1}(t)+\left[1-P_{j-1}(t)\right] \pi_{u}(t)
$$

en donde

$$
\pi_{u}(t)=\frac{\delta[1-d(t)]}{d(t)}
$$

y donde

$\pi_{u}(t)=$ probabilidad de que un desempleado en el medio urbano encuentre trabajo urbano;

$\delta=$ tasa de crecimiento de la población empleada en el área metropolitana durante el período 1950-1970;

$d(t)=$ tasa de desempleo en el área metropolitana en el año $t$ durante el período 1950-1970.

Por inducción se puede demostrar que

$P_{j}(t)=1-[1-\pi(t)]$, es decir, $P_{j}(t)$ crece exponencialmente con el tiempo de "búsqueda" $j$. 
en la diferencia de los ingresos rural y urbano duplicaría la corriente migratoria al área metropolitana de la ciudad de México.

Debe hacerse notar que el valor de $R^{2}$ se reduce al introducir la probabilidad de encontrar empleo urbano. La explicación de esto puede radicar en el supuesto, utilizado hasta ahora, de que en el medio rural hay pleno empleo y, por tanto, que el ingreso esperado en las áreas rurales coincide con el ingreso derivado del salario mínimo en ellas.

Se modifica ahora este supuesto a todas luces irreal y se supone que el ingreso esperado en el medio rural es igual a $\pi_{R} Y_{R},\left(Y_{R}=\right.$ ingreso de salario mínimo rural y; $\pi_{R}=$ la probabilidad de obtener empleo-con la remuneración de salario mínimo rural) ${ }_{0}^{3}$ se tiene que la diferencia de ingreso está dada ahora por:

$$
\begin{aligned}
& \alpha_{4}(t)=Y_{u}-Y_{R} \pi_{R} \\
& \alpha_{5}(t)=Y_{u} \pi_{u}-Y_{R} \pi_{R}
\end{aligned}
$$

en donde:

$\alpha_{4}(t), \alpha_{s}(t)=$ diferencia de ingreso rural-urbano;

$Y_{u}, Y_{R}=$ ingresos anuales del medio urbano y del rural respectivamente;

$\pi_{u}, \pi_{R}=$ probabilidades de encontrar empleo en el medio urbano y el rural, respectivamente.

Los resultados que se obtuvieron según esta especificación son:

$$
\begin{aligned}
L M= & 4.87+0.649 L \alpha_{4}(t) \\
& (0.266) \\
R^{2}= & 0.426 ; F=12.6 ; D . W .=2.01 \\
\mathrm{LM}= & 5.92+0.512 L \alpha_{5}(t) \\
\quad(0.155) & \\
R^{2}= & 0.494 ; F=16.57 ; D . W .=2.03
\end{aligned}
$$

En las regresiones 28 y 29 , se puede observar que aun después de abandonar el supuesto de empleo pleno rural e introducir un elemento de rechazo de las áreas rurales, representado por la existencia de desempleo rural, la diferencia del ingreso rural-urbano afecta de manera considerable la migración. Según estas ecuaciones, un aumento de, por ejemplo, $100 \%$ de la diferencia entre los ingresos del área metropolitana de la ciudad de México y los rurales, aumenta en $65 \%$ según la regresión 28 y $50 \%$ según la regresión 29 .

3 Los valores de $\pi_{R}$ se obtuvieron de los cálculos de desempleo en el medio rural para 1950-1970 según Manuel Gollás (1972). 
Estos últimos resultados muestran que al introducir el concepto de probabilidad de encontrar empleo en el medio urbano y rural hay una pequeña mejora en la explicación de la migración. ${ }^{*}$ En las ecuaciones semilogarítmicas, en las deficiones de $\alpha_{4}(t)$ y $\alpha_{5}(t), R^{2}$ pasa de un valor de 0.426 a uno de 0.494 (regresiones 28 y 29 ).

En este caso las elasticidades de migración respecto a la diferencia de ingreso y respecto a la probabilidad de encontrar empleo son respectivamente:

$$
\begin{aligned}
& \epsilon_{M}, \alpha_{5}(t)=0.512 \\
& \epsilon_{M}, \pi_{u}=\epsilon_{M} \alpha_{5} \times \epsilon \alpha_{5}, \pi_{u} \\
& \text { para 1950: } \epsilon_{M}, \pi_{u}=0.552 \\
& \text { para 1959: } \epsilon_{M}, \pi_{u}=0.533 \\
& \text { para 1969: } \epsilon_{M}, \pi_{u}=0.530
\end{aligned}
$$

Se puede afirmar que, según estos cálculos, tanto la diferencia de ingresos rural-urbana como la creación de empleo urbano estimulan la migración. En ambos casos se observa una elasticidad de la migración al área metropolitana de la ciudad de México de alrededor de 0.5.

$\mathrm{Si}$ ahora se considera que la diferencia de ingreso rural-urbana es entre flujos esperados y se sigue suponiendo que hay desempleo urbano y rural $^{5}$ se tiene que:

$$
\begin{aligned}
& \alpha_{\theta}(t)=\sum_{j=1}^{N}-\frac{Y_{u j}(t)-\pi_{R}(t) \bar{Y}_{j}(t)}{(1+r)^{j}} \\
& \alpha_{i}(t)=\sum_{j=1}^{N} \frac{Y_{u j}(t) P_{j}(t)-\pi_{R}(t) Y_{j}(t)}{(1+r)^{j}}
\end{aligned}
$$

Los resultados obtenidos según esta formulación son:

4 Según otros estudios (Barnum y Sabot, 1975), el aumento en el valor de $R^{2}$ al introducir la probabilidad de empleo es también pequeño. En este estudio $R^{2}$ aumenta de 0.48 a 0.55 .

5 Recuérdese el significado de $\pi_{u}(t)$ y $P_{j}(t)$ es diferente. $\pi_{u}(t)$ es la probabilidad de que un desempleado en el medio urbano encuentre trabajo $y$, por otra parte, $P_{j}(t)$ es la probabilidad de que un migrante que llegó al medio urbano en el año $t$ esté trabajando en el año $t+j$.

Por otra parte, $\pi_{u}(t)$ puede expresarse como sigue:

$$
\pi_{u}(t)=\frac{\delta N_{t}}{S_{t}-N_{t}}
$$

en donde:

$\delta=$ tasa de crecimiento de la población urbana empleada;

$N_{t}=$ población empleada;

$S_{t}=$ fuerza de trabajo como se explicó antes $P_{j}(t)$ es función de $\pi_{u}(t)$. 


$$
\begin{aligned}
& L M= 8.895+0.0015 \alpha_{6}(t) \\
&(0.00033) \\
& R^{2}=0.576 ; F=23.1 ; D . W .=2.03 \\
& L M=-6.4+1.468 L \alpha_{6}(t) \\
& \quad(0.336) \\
& R^{2}=0.567 ; F=21.7 ; D . W .=2.06 \\
& L M=8.7+0.0017 \alpha_{7}(t) \\
& \quad(0.00038) \\
& R^{2}=0.578 ; F=23.28 ; D . W .=2.03 \\
& L M=-8.318+1.634 L \alpha_{7}(t) \\
& \quad(0.382) \\
& R^{2}=0.562 ; F=21.2 ; D . W .+2.07
\end{aligned}
$$

En las ecuaciones con la definición de la variable $\alpha_{6}(t)$ y $\alpha_{7}(t)$ hay un comportamiento diferente entre la especificación semilogarítmica y la doblelogarítmica. En la primera hay un aumento para $R^{2}$ de 0.576 a 0.578 , al incorporar la probabilidad de empleo mientras que en la doblelogarítmica hay una disminución de 0.567 a 0.562 .

La elasticidad de migración respecto a la diferencia de ingreso en este caso es:

$$
\epsilon_{M}, \alpha_{7}(t)=1.63
$$

La elasticidad de migración con respecto a la probabilidad de encontrar empleo es:

$$
\begin{aligned}
& \epsilon_{M}, \pi_{u}=\epsilon_{M}, \alpha_{7}(t) \epsilon_{7}(t), \pi_{u} \\
& \text { para 1959: } \epsilon_{M}, \pi_{u}=0.0517 \\
& \text { para 1969: } \epsilon_{M}, \pi_{u}=0.098
\end{aligned}
$$

De acuerdo con los resultados obtenidos de la definición $\alpha_{7}(t)$ conforme a la cual la diferencia de ingreso rural-urbano se identifica como una diferencia de flujos y se tiene en cuenta la probabilidad de empleo urbano y rural, tanto la diferencia de ingresos como la creación de empleo urbano influyen en la migración. Sin embargo, debe observarse que la migración, según estos cálculos, responde más a cambios en la diferencia de ingresos rural-urbana $\left(\epsilon_{M}, \alpha_{\tau}(t)=1.63\right)$ que a cambios en la disponibilidad de empleos urbanos:

$$
\left(\epsilon_{M}, \pi_{u}=0.0517 \mathrm{y} \epsilon_{M}, \pi_{u}=0.098\right)
$$

\section{Análisis de corte transversal}

En este estudio también se hicieron pruebas de corte transversal para verificar las hipótesis sobre la importancia que tienen para la migración 
tanto la diferencia de ingresos urbanos y rurales como el desempleo urbano.

De acuerdo con esta formulación se tiene que:

$$
\begin{aligned}
& M Q 4=M(\alpha, Q 4, S Q 4) \\
& T M Q 4=M(\alpha, Q 4, S Q 4)
\end{aligned}
$$

en donde

$M Q 4=$ número de migrantes para el 4o. quinquenio del período $1955-$ 1969 por entidad federativa;

$T M Q 4=$ cociente migratorio definido como:

$$
T M Q 4=\frac{M Q 4}{P}
$$

$(P=$ población del área metropolitana en el año central del 4o. quinquenio, o sea 1968);

$\alpha_{1} Q 4=$ diferencia de ingreso rural-urbano para el 4o. quinquenio por entidad federativa y según definición de la ecuación (12);

$S Q 4=$ la tasa de subempleo para cada entidad federativa.

Las ecuaciones de regresión que se obtuvieron son:

$$
\begin{aligned}
& L M Q 4=-1.424+1.078 L \alpha_{1} Q 4+3.37 S Q 4 \\
& (0.574) \quad(1.98) \\
& R^{2}(L \alpha)=0.204 ; F(1,29)=7.41 \\
& R^{2}(L \alpha ; S Q)=0.278 ; F(2,28)=5.4 \\
& L T M Q 4=-9.978+0.496 L \alpha_{1} Q 4+3.57 S Q 4 \\
& \quad(0.299) \quad(1.029) \\
& R^{2}(S Q)=0.401 ; F(1,29)=19.4 \\
& R^{2}(S Q, L \alpha)=0.454 ; F(2,28)=11.7
\end{aligned}
$$

La explicación de la migración resulta mejor cuando se considera el cociente de migración en lugar del volumen de migración: $R^{2}$ aumenta de 0.278 a 0.454 . Sin embargo, en la ecuación (39) la variable que tiene un poder explicativo mayor es la que corresponde al indicador de subempleo en el lugar de origen.

Ahora bien, el bajo poder explicativo de la variable diferencial de ingreso en las ecuaciones de corte transversal introduce un elemento de duda sobre el poder explicativo de esta variable en la migración. 
Las elasticidades que se encuentran a partir de estas ecuaciones son:

$$
\epsilon_{M}, \alpha Q 4=1.078 ; \epsilon_{T M}, \alpha Q 4=0.496
$$

Las interpretaciones de estas elasticidades son similares a las anteriores.

\section{RESUMEN}

Según los resultados de este estudio, la diferencia de ingreso rural urbano que se calcula teniendo en cuenta el desempleo rural y urbano, explica la migración rural al área metropolitana de la ciudad de México. Por su parte, el número de empleos urbanos disponibles (la probabilidad de encontrar empleo urbano) es también importante para explicar la migración, aunque menos que la diferencia de ingreso. Dicho en otra forma, un aumento porcentual en la diferencia entre el ingreso rural y el urbano estimula más la migración que un aumento porcentual similar en el número de empleos urbanos.

Por último, el análisis apoya la tesis de que los programas para crear más empleos urbanos o aumentar los ingresos urbanos tienen como resultado acentuar la migración rural urbana y agravar el desempleo urbano. Las soluciones al desempleo urbano se encuentran no en la creación de empleo urbano sino en la creación de empleo rural.

\section{APENDICE}

\section{Salario mínimo}

En el cálculo de los ingresos esperados por el migrante tanto para el medio urbano como para el rural se trabajó con la información de salario mínimo. ${ }^{1}$ Es la única información sobre ingreso que abarca todos los años del período 1950-1970 a nivel regional.

Su utilización tiene ciertas limitaciones importantes que conviene destacar y analizar.

1) En primer lugar, la información sobre salario mínimo se presenta en forma nominal y es a partir de 1965 cuando la CNSM agrega los resultados de la elaboración de un índice de precios acorde con los criterios de fijación del salario mínimo.

La variable diferencial de ingreso utilizada en todas las regresiones de serie de tiempo se presenta en forma nominal. Los intentos de extrapolar retrospectivamente dicho índice de precios no resultaron satisfactorios. La única opción que resta es la de utilizar un índice de precios que

\footnotetext{
1 Anuarios de la Comisión Nacional de Salarios Mínimos.
} 
abarque todo el período; este índice es el del Banco de México para los preciós al mayoreo en la ciudad de México; ${ }^{2}$ la restricción es que, al usarlo, se está considerando que no hubo discrepancias en los movimientos de los precios entre el área metropolitana y el medio rural del país.

En el cuadro 1 se muestran los niveles de ingreso anual de salario mínimo para el AM y para el medio ural $^{3}$ en términos nominales y reales. Estos últimos se obtuvieron al deflacionar con el índice de precios al mayoreo elaborado por el Banco de México. Se observa que el salario mínimo deflacionado presenta un crecimiento importante $(7.2 \%$ anual para el medio urbano y $5.9 \%$ anual para el medio rural).

2) Dado que en todas las pruebas se ha identificado el ingreso esperado por el migrante con el ingreso correspondiente al salario mínimo, es muy importante tener una idea de la bondad de dicha identificación.

Ahora bien, la CNSM no dispone de información respecto a la vigencia del salario mínimo en las zonas establecidas legalmente y no hay otra fuente que nos proporcione información sobre ingreso por períodos bianuales.

\section{Cuadro 1}

INGReso anUal por salario mínimo para el ĀRea Metropolitana DE LA CIUDAD DE MÉXICO E INGRESO MEDIO PARA EL MEDIO RURAL, EN TÉRMINOS NOMINALES Y REALES, 1950-1969

\begin{tabular}{|c|c|c|c|c|c|c|}
\hline \multirow{2}{*}{ Aros } & \multicolumn{3}{|c|}{$\begin{array}{l}\text { Area Metropolitana de la } \\
\text { Ciudad de Mexico }\end{array}$} & \multicolumn{2}{|c|}{ Medio rural } & \multirow{2}{*}{ Indtoe } \\
\hline & Nominal & & Real & Nominal & Real & \\
\hline $\begin{array}{l}1950 \\
1951\end{array}$ & 1237.35 & 1 & 706.69 & 965.79 & 1332.12 & $\begin{array}{l}0.725 \\
0.898\end{array}$ \\
\hline $\begin{array}{l}1952 \\
1953\end{array}$ & 2445.5 & 2 & 623.93 & 1300.00 & 1399.85 & $\begin{array}{l}0.932 \\
0.914\end{array}$ \\
\hline $\begin{array}{l}1954 \\
1955\end{array}$ & 2920.0 & 2 & 920.0 & 1785.58 & 1785.58 & $\begin{array}{l}1.000 \\
1.138\end{array}$ \\
\hline $\begin{array}{l}1956 \\
1957\end{array}$ & 4015.0 & 3 & 376.79 & 2050.20 & 1724.31 & $\begin{array}{l}1.189 \\
1.240\end{array}$ \\
\hline $\begin{array}{l}1958 \\
1959\end{array}$ & 4380.0 & 3 & 382.24 & 2354.25 & 1817.95 & $\begin{array}{l}1.295 \\
1.310\end{array}$ \\
\hline $\begin{array}{l}1960 \\
1961\end{array}$ & 5292.6 & 3 & 849.09 & 2868.17 & 2085.94 & $\begin{array}{l}1375 \\
1.388\end{array}$ \\
\hline $\begin{array}{l}1962 \\
1963\end{array}$ & 6387.5 & 4 & 520.52 & 3519.69 & 2490.93 & $\begin{array}{l}1.413 \\
1.421 .\end{array}$ \\
\hline $\begin{array}{l}1964 \\
1965\end{array}$ & 7847.5 & 5 & 298.78 & 4676.74 & 3517.83 & $\begin{array}{l}1.481 \\
1.508\end{array}$ \\
\hline $\begin{array}{l}1966 \\
1967\end{array}$ & 9125.0 & 5 & 971.86 & 5489.60 & 3592.67 & $\begin{array}{l}1.528 \\
1.572\end{array}$ \\
\hline $\begin{array}{l}1868 \\
1969\end{array}$ & $10 \quad 811.25$ & 6 & 436.49 & B 398.45 & 3994.04 & $\begin{array}{l}1.802 \\
1.642\end{array}$ \\
\hline
\end{tabular}

FuEnte: Anuario de la Comisión Nacional de Salarios Mínimos; e Informe Anual, Banco de México.

2 “Indicadores Económicos", Banco de México.

${ }^{3}$ El ingreso del medio rural es la media aritmética simple de los ingresos correspondientes a los estados de Aguascalientes, Campeche, Colima, Chiapas, Guanajuato, Guerrero, Hidalgo, Jalisco, México, Michoacán, Morelos, Nayarit, Oaxaca, Puebla. Querétaro, Quintana Roo, San Luis Potosí, Tabasco, Tlaxcala, Veracruz, Yucatán y Zacatecas. De estos estados proviene el $90 \%$ de los migrantes. 
Sin embargo, es posible, hacer comparaciones con la información censal y con las encuestas de ingreso-gasto.

La comparación realizada entre el ingreso medio de salario mínimo y el ingreso medio calculado a partir del censo de 1970 muestra variaciones importantes entre entidades en cuanto a la vigencia del salario mínimo.

En las pruebas que se efectuaron con información de corte transversal, donde se incluyó como segunda variable explicativa una "tasa de subempleo" por entidad federativa, la cual se calculó considerando como subempleo a todos aquellos que declararon en el censo de 1970 un ingreso menor al correspondiente del salario mínimo, se puede apreciar que el efecto de las variaciones estatales en la vigencia del salario mínimo es significativo.

\section{Empleo}

La fuente de información utilizada para la elaboración de la variable diferencial de ingreso que incorpora la probabilidad de encontrar empleo en el AM, es la Encuesta Continua de Mano de Obra. Esta encuesta proporciona la información sobre índices de desempleo trimestralmente para los años 1972 y siguientes para el área metropolitana.

Por interpolación retrospectiva se obtienen los índices de desempleo para los años comprendidos entre 1950 y 1969 . También a partir de un ajuste de la población empleada se obtiene su tasa media de crecimiento anual. (Se utilizó como base la magnitud de población empleada dada en el censo de 1950.)

La curva ajustada para la población empleada es:

$N(t)=(732340) 60.034$

$(t=1)(1950) ; t=2(1951) ; t=24$ (1973)

La tasa de crecimiento es $\gamma=0.034$

La curva ajustada para el índice de desempleo es $D(t)=100-94 b-0.00157 t$

Comparación entre las magnitudes calculadas por este procedimiento y las correspondientes dadas por el censo.

\begin{tabular}{cccccc}
\hline & \multicolumn{2}{c}{ Población empleada } & & \multicolumn{2}{c}{ Indice de desempleo } \\
\cline { 2 - 3 } \cline { 5 - 6 } & Ajuste & Censo & & Ajuste & Censo \\
\hline 1950 & 757668 & 757460 & 2.7 & 2.5 \\
1960 & 1064483 & 1183946 & & 4.21 & 2.9 \\
1970 & 1495543 & 1435659 & 5.7 & 4.28 \\
\hline
\end{tabular}


El procedimiento anterior tiene dos limitaciones serias:

a) Los valores obtenidos como índice de desempleo corresponden al desempleo abierto. En el modelo utilizado, lo que se requiere para calcular $I I(t)$ es un índice de subempleo que distinga el "sector moderno" del "sector urbano tradicional". No hay información disponible al respecto.

Ahora bien, es por esta deficiencia que los valores de $I I(t)$ calculados muestran, de 1950 a 1958 , un valor igual a la unidad y de ahí en adelante bajan hasta un valor de 0.676 .

b) No fue posible encontrar información sobre las variaciones en los niveles de desempleo para los años del período 1950-1970, la cual hubiera podido ser utilizada para explicar variaciones en el volumen migratorio.

\section{BIBLIOGRAFIA}

ANUARIO, Comisión Nacional de Salarios Mínimos, Secretaría del Trabajo, México, varios años.

Barnum, H. y R. H. Sabot, "Education Employment Probabilities and Rural Urban Migration in Tanzania", International Bank for Reconstruction and Development, 1975.

Benítez Zenteno, Raúl, y Gustavo Cabrera, Proyecciones de la población de México. 1960-1980, Banco de México, 1966.

Bowles, S., "Migrations as an Investment: Emperical Test of the Human Investment Approach" en Review of Economics and Statistics, noviembre de 1970.

Courchene, T. J., "Interprovincial Migration and Economic Adjustment", Canadian Journal of Economics, 3, noviembre de 1970, 550-576.

De Oliveira Orlandina, Brígida García y Humberto Muñoz, "Migraciones Internas y grupos populares urbanos: Ciudad de México (1950-1970)"; Revista Mexicana de Sociología, Núm. 1, Año 38, 1979.

Fabricant, R. A., "An Expectational Model of Migration", Journal of Regional Science, 10, abril de 1970, 13-24.

Gallaway, L. E., R. F. Gilbert y P. E. Smith, "The Economics of Labor Mobility: An Empirical Analysis, Western Economic Journal, 1967, 211-223.

Gollás Manuel, "El desempleo agrícola en México", en Edmundo Flores (Comp.), Desarrollo agrícola, México, Fondo de Cultura Económica, 1972.

Greenwood, M. J., "An Analysis of the Determinants of Geographic Labor Mobility in the United States", Review of Economics and Statistics, mayo de 1966.

Gugler, J., "On The Theory of Rural-Urban Migration: The case of SubSaharan Africa" en J. A. Jackson (Comp.), Sociological Studies Two: Migration, Cambridge University Press, 1969. 
Herrick, B., Urban Migration and Economic Migration in Chile, Cambridge, M.I.T. Press, 1965.

Indicadores Económicos, Banco de México, México, varios Jaños.

Informe anual, México, Banco de México, varios años.

Isbister, John W., "The Growth of Employment in Mexico", Tesis doctoral, Princeton University, 1969.

Laber, G. y R. X. Chase, "Interprovincial Migration in Canada as a Human Capital Decision, Journal of Political Economy, junio-agosto de 1971, 795-804.

Lowry, I. S., "Migration and Metropolitan Growth: Two Analytical Models", Los Angeles, Institute of Government and Public Affairs, UCLA, 1966.

Miller, E., "Is Out-Migration Affected by Economic Conditions?", Southern Economic Journal, 29, enero de 1973, 396-405.

Muñoz Humberto, Orlandina de Oliveira y Claudio Stern, Migración y desigualdad social en la ciudad de México, México, El Colegio de México, 1979.

Reynols, Lloyd y Peter Gregory, Wages, Productivity and Industrialization in Puerto Rico, Homewood, Illinois, Richard D. Irwin, Inc., 1965.

Rogers, A., "A Regression Analysis of Interregional Migration in California, Review of Economics and Statistics, mayo de 1967.

Sahota, G. S., "An Economic Analysis of Internal Migration in Brazil", Journal of Political Economy, mayo de 1968.

Schultz, T. P., "Rural-Urban Migration in Colombia" en Review of Economics and Statistics, mayo de 1971.

Sjaastad, L. A., "The Cost and Returns of Human Migration" en Journal of Political Economy, octubre de 1962.

Sommers, P. M. y D. B. Suits, "Analysis of Net Interstate Migration", Southern Economic Journal, 40, octubre de 1973, 193-201.

Stoltman, Joseph y John M. Ball, "Migration and The Logical Economic Factor in Rural Mexico", Human Organization, 1971.

Stouffer, Samuel, "Intervening Opportunities: A Theory Relating Mobility and Distance", en American Sociological Review, 1940.

Todaro, M. P., "A Model of Labor Migration and Urban Unemployment in Less Developed Countries" en American Economic Review, marzo de 1969.

Todaro, M. P., "Income Expectations, Rural-Urban Migration and Employment in Africa", International Labor Review, noviembre de 1971.

Trejo, Saúl, Industrialización y empleo en México, México, Fondo de Cultura Económica, 1978.

Wadycki, W. J., "Alternative Opportunities and Interstate Migration: Some Additional Results", Review of Economics and Statistics, mayo de 1974. 
Whetten, Nathan y R., Burnight, "Internal Migration in Mexico", Rural Sociology, Vol. 21, junio de 1956.

Zipf, George K., "The $\mathrm{P}_{1} \mathrm{P}_{2} / \mathrm{O}$ Hypotesis: On the Intercity Movement of Persons", American Sociological Review, 1946. 